\title{
IMAGE QUALITY IMPROVEMENTS IN LOW-COST UNDERWATER PHOTOGRAMMETRY
}

\author{
F. Neyer ${ }^{1,2^{*}}$, E. Nocerino ${ }^{1,3}$, A. Gruen ${ }^{1}$
}

${ }^{1}$ Institute of Theoretical Physics, ETH Zurich, 8093 Zurich, Switzerland - (neyerf, agruen)@ geod.baug.ethz.ch, erican@phys.ethz.ch

${ }^{2}$ Institute of Geodesy and Photogrammetry, ETH Zurich, 8093 Zurich, Switzerland

${ }^{3}$ Aix-Marseille Université, CNRS, ENSAM, Université De Toulon, LIS UMR 7020, Domaine Universitaire de Saint-Jérôme, Bâtiment Polytech, Avenue Escadrille Normandie-Niemen, 13397, Marseille, France - erica.nocerino@univ-amu.fr

\section{Commission II, WG II/9}

KEY WORDS: Underwater photogrammetry, image quality, action cameras, GoPro, accuracy evaluation, 3D modelling

\begin{abstract}
This study presents an evaluation of a cheap consumer-grade camera used for modelling a coral reef section. We evaluate the quality of a reconstructed coral reef using GoPro cameras and a high-end camera with data from an actual coral reef dataset. We also investigate components of the processing pipeline (like image quality) separate from the final results. Because our GoPro images suffer from severe chromatic aberration, we apply different image pre-processing steps to improve their quality and show its effects on the reconstructed object points. Bundle adjustment is carried out as free networks in all cases, with a follow-up rigid 3D Helmert transformation onto a geodetic control network, carried out to define the common datum and to remove the bias from the free network results.
\end{abstract}

\section{INTRODUCTION}

Underwater photogrammetry is a popular and relatively cheap method for modelling underwater areas at different scales and at different levels of accuracy. One of the reasons this technique is used for various applications is the quality of camera sensors and their optical components (lenses and domes) that has rapidly improved over the last years. While DSLR or high-end mirrorless cameras are the typical choices for demanding tasks, the quality of video cameras has increased accordingly and therefore might also be an option for underwater reconstruction tasks. Action cameras like GoPro or Sony that are equipped with underwater protection covers are cheap alternatives to high-end cameras like full-frame DSLR cameras with dedicated underwater housings. While action cameras typically have a considerably smaller entrance pupil, smaller pixel dimensions (i.e., $1.55 \mu \mathrm{m}$ for GoPro Hero 7), and a flat dome port, dedicated underwater housings with DSLR cameras chosen for optimal performance under lowlight conditions seem to be better suited for the task of highaccuracy underwater modelling. In a previous study the accuracy performance of GoPro and Lumix cameras were compared in air and underwater (Guo et al., 2016). This was done by comparing the final results of a controlled object reconstruction by using a calibration frame with signalized points. Some problematic aspects when dealing with high accuracy underwater control point frames where addressed in Neyer et al. (2018).

Our study site is located in Moorea, Tahiti, French Polynesia. It is part of the Moorea Island Digital Ecosystem Avatar (IDEA) project (https://mooreaidea.ethz.ch/) with an international team of researchers. While the IDEA project includes many aspects of digitizing the whole island ecosystem, our task and ultimate goal here is to provide an easy-to-use procedure for underwater coral reef change detection at the $\mathrm{cm}$ to $\mathrm{mm}$ scale.

In our evaluation of GoPro image quality improvements, we use a high-end camera (Panasonic Lumix GH4) as reference. All measurements were acquired in August 2018.

\section{METHODS}

\subsection{Reference Frame}

For change detection, a control network was set up at one of our test sites (5 x $5 \mathrm{~m}$, Figure 1a). The control network was established using a dedicated construction of aluminum targets, anchored into dead corals or rocks. We use coded targets for automatic detection of the corresponding image coordinates in our datasets. For estimating the object coordinates in a local reference frame, multiple distance and leveling measurements (with a green light laser pointer) were used as raw observations. Applying the principles of trilateration, the geodetic network was optimized using Trinet+ software (Guillaume et al., 2008) as a free network. Careful evaluation yielded accuracy in the order of $1.5 \mathrm{~mm}$ for all components. Details of the procedure are given in Neyer et al. (2018) and Nocerino et al. (2019).

The geodetic reference frame is used to anchor the different models such that comparisons can be carried out without any additional alignment step of the dense photogrammetric point clouds. 


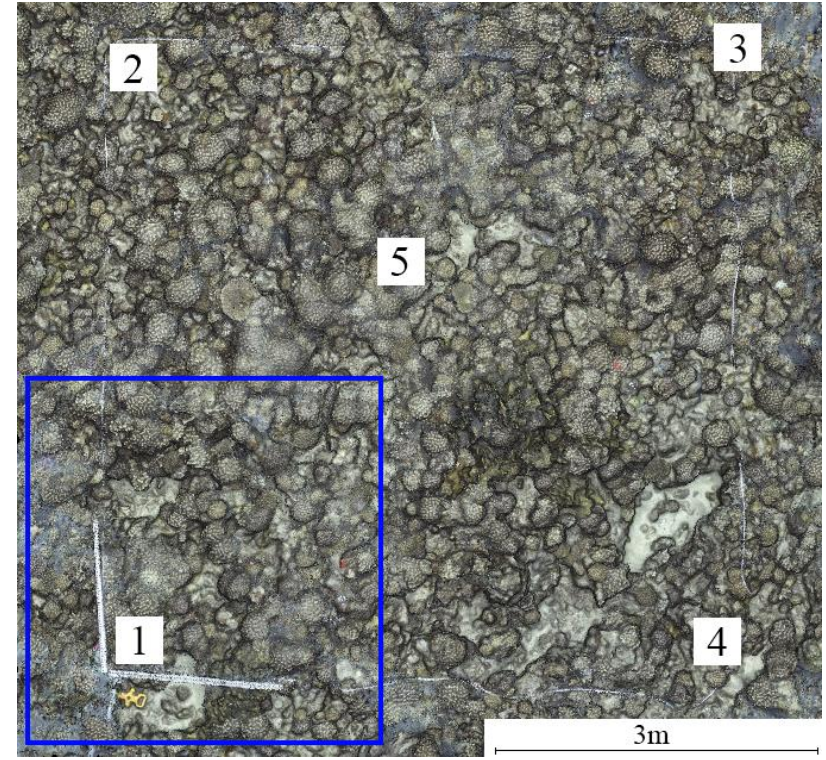

Figure 1. Coral testfield no. 18 (roughly 5 x 5 meter within the control points marked from 1 to 5 ). The blue rectangle highlights the section (roughly $2 \times 2 \mathrm{~m}$ ) were we extracted two small areas used for comparing the dense point clouds of the different correction models.

\subsection{Image preparation}

The two principle image datasets used in this study are images taken with a GoPro camera (with an average GSD of $1.2 \mathrm{~mm}$ ) and a Panasonic Lumix GH4 camera (average GSD of $0.6 \mathrm{~mm}$ ), furthermore named reference data. An experienced diver acquired both datasets of the $5 \times 5 \mathrm{~m}$ test field, one after the other. In both cases, the cameras were pointing in nadir direction. Table 1 summarizes the acquisition details.

\begin{tabular}{|c|c|c|c|}
\hline & $\begin{array}{c}\text { acquisition } \\
\text { mode }\end{array}$ & \# images & $\begin{array}{c}\text { white } \\
\text { balance }\end{array}$ \\
\hline GoPro & video & $\begin{array}{c}431 \\
\text { (extracted) }\end{array}$ & red filter \\
\hline Lumix & raw images & 451 & $\begin{array}{c}\text { digitally } \\
\text { (global) }\end{array}$ \\
\hline
\end{tabular}

Table 1. GoPro and Lumix dataset acquisition details. GoPro images were extracted from the video sequence at a constant time rate

Besides the difference in GSD, obvious image quality differences are visible (Figure 2): GoPro images suffer from

- $\quad$ severe chromatic aberration

- image compression artifacts (visible in a full resolution)

- $\quad$ lack of contrast in some areas

Because the reference images were stored as RAW files, a global white balance adjustment could be applied digitally before converting the images to the jpg format.
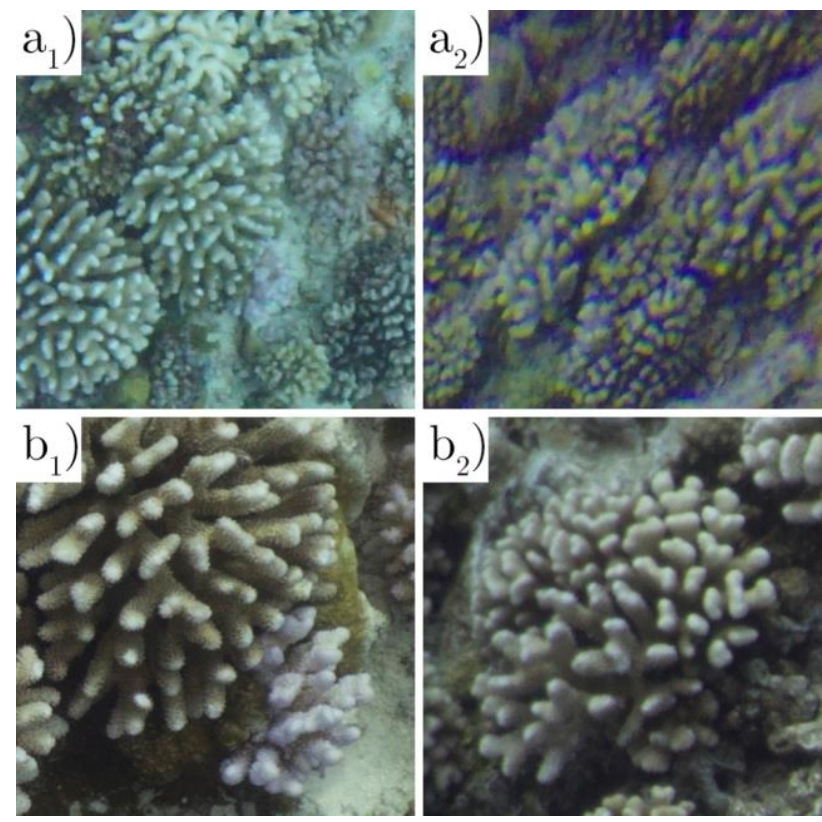

Figure 2. Sections of example images of a) GoPro and b) Lumix GH4 cameras. The left column (subscript 1) shows the image quality at the center of the frame and on the right column (subscript 2), at the very corner of the frame.

\subsection{Reduction of Chromatic Aberration}

Image quality in GoPro cameras is most severely affected by chromatic aberration (CA) and a blurred projection increasing further away from the image center (Figure 2a). Blurred image parts cannot be fully recovered and as such we only concentrate on the improvement of CA. For the pure aim of bundle adjustment and 3D modelling, a single color channel can be used to overcome this error. Usually, however, color is an important information (for example for classification) and generally contains more structural information for the matching process than a single color channel can provide. CA correction can in principle be conducted by an individual calibration of the different color channels. Images can thereafter be undistorted channel-wise and recombined to a full RGB image. An alternative is to correct two color channels with respect to the third. Because the latter option is more flexible, i.e., an independent CA correction model can be applied, we chose to align the red and blue channels with respect to the green. This task involves three steps:

(1) Displacements of the red and blue channels with respect to the green channel are estimated.

(2) A correction model is defined. The displacements between the channels are used to estimate the parameters (an independent set of parameters for the red and blue channel):

a) Brown model, Brown (1971)

b) Collocation model, Moritz (1973)

(3) The red and blue channels are corrected and the color image is re-build.

Steps (1), (2a) and (2b) are explained in more detail in the following sections. 


\section{Estimation of relative displacements}

Relative displacements among the channels are estimated using an optical flow procedure introduced by Farneback (2003). Pixel displacements from red and blue with respect to the green channel are estimated for all images in the survey (431). In a next step, the median displacements for all image coordinates are taken for both the red and blue channel. This dataset is then reduced to 4000 uniformly distributed measurement coordinates for both channels. More measurements were not necessary to reliably estimated the parameters of the different correction models.

\section{Brown model}

The well-known camera calibration model (Brown, 1971) with two shift $\left(x_{p}, y_{p}\right)$, three radial distortion $\left(k_{1,2,3}\right)$, and two tangential distortion $\left(p_{1,2}\right)$, parameters is given by

$$
\begin{aligned}
& x=x_{0}(1+d r)+d t_{x} \\
& y=y_{0}(1+d r)+d t_{y}
\end{aligned}
$$

with

$$
\begin{aligned}
d r & =k_{1} r^{2}+k_{2} r^{4}+k_{3} r^{6} \\
d t_{x} & =p_{1}\left(r^{2}+2 x_{0}^{2}\right)+2 p_{2} x_{0} y_{0} \\
d t_{y} & =p_{2}\left(r^{2}+2 y_{0}^{2}\right)+2 p_{1} x_{0} y_{0} \\
r^{2} & =x_{0}^{2}+y_{0}^{2} \\
x_{0} & =x_{d}-x_{p} \\
y_{0} & =y_{d}-y_{p}
\end{aligned}
$$

Whereas $(x, y)$ are the undistorted image coordinates and $\left(x_{d}, y_{d}\right)$ the distorted image coordinates.

\section{Collocation}

Least-squares collocation is a well-known method for the differentiation between measurement noise and signal based on assigned neighborhood relations (i.e., correlations). In the geodetic context, this procedure is well described in Moritz, 1970 and Moritz, 1973. A linear estimator, in our case the displacements at the different image coordinates, $\boldsymbol{d}(\boldsymbol{x}, \boldsymbol{y})$ is combined with an empirical estimate of correlations in a stochastic field $\boldsymbol{C}_{\boldsymbol{s} \boldsymbol{s}}$. If the noise component $\boldsymbol{n}$ can be assumed to be uncorrelated with the signal $\boldsymbol{s}$ and $\boldsymbol{s} \sim \mathcal{N}\left(0 ; \boldsymbol{C}_{\boldsymbol{s} s}\right)$ and $\boldsymbol{n} \sim \mathcal{N}\left(0 ; \boldsymbol{C}_{\boldsymbol{n} \boldsymbol{n}}\right)$, the following solution can be formulated:

$$
\begin{aligned}
\boldsymbol{d}(\boldsymbol{x}, \boldsymbol{y}) & =\boldsymbol{A} \widehat{\boldsymbol{p}}+\widehat{\boldsymbol{s}}+\widehat{\boldsymbol{n}} \\
\boldsymbol{D} & =\left(\boldsymbol{C}_{\boldsymbol{s}}+\boldsymbol{C}_{\boldsymbol{n} n}\right)^{-1} \\
\widehat{\boldsymbol{k}} & =\boldsymbol{D} \cdot(\boldsymbol{d}(x, y)-\boldsymbol{A} \widehat{\boldsymbol{x}}) \\
\widehat{\boldsymbol{s}} & =\boldsymbol{C}_{\boldsymbol{s}} \cdot \widehat{\boldsymbol{k}} \\
\widehat{\boldsymbol{n}} & =\boldsymbol{C}_{\boldsymbol{n} \boldsymbol{n}} \cdot \widehat{\boldsymbol{k}}
\end{aligned}
$$

with $(\hat{.})$ indicating an estimated component and $\boldsymbol{A}$ being a deterministic model. The least-squares system minimizes $\boldsymbol{s}^{T} \boldsymbol{C}_{\boldsymbol{s}}^{-1} \boldsymbol{s}+\boldsymbol{n}^{T} \boldsymbol{C}_{\boldsymbol{n} \boldsymbol{n}}^{-1} \boldsymbol{n}$. More details can be found in Neyer, 2016.

The solution in (2) can be computed once the deterministic model $\boldsymbol{A}$ and the stochastic matrices $\boldsymbol{C}_{\boldsymbol{s} \boldsymbol{s}}$ and $\boldsymbol{C}_{\boldsymbol{n} \boldsymbol{n}}$ are defined. $\boldsymbol{A}$ in its most primitive form may be chosen as simple mean to centralize the measurements (required due to $\boldsymbol{s} \sim \mathcal{N}\left(0 ; \boldsymbol{C}_{\boldsymbol{s} s}\right)$ and $\left.\boldsymbol{n} \sim \mathcal{N}\left(0 ; \boldsymbol{C}_{\boldsymbol{n} \boldsymbol{n}}\right)\right)$. Here we chose a polynomial of $2^{\text {nd }}$ order. The choice of the deterministic model is not critical here as we have continuous dense measurements. $\boldsymbol{C}_{\boldsymbol{n} n}$ is a diagonal matrix (i.e., no correlations between noise) and equal for all displacement measurements. $\boldsymbol{C}_{\boldsymbol{s} \boldsymbol{s}}$, the correlation matrix, is empirically estimated using the following model (using $\boldsymbol{r}$ as the position vector):

$$
\begin{aligned}
\boldsymbol{f}\left(\boldsymbol{r}_{i j}\right) & =\boldsymbol{\sigma}_{s}^{2} \cdot \boldsymbol{e}^{-u \cdot\left|\boldsymbol{r}_{i}-\boldsymbol{r}_{j}\right|^{n}} \\
\boldsymbol{r}_{s} & =\left(\frac{-\ln (\mathbf{0 . 5})}{u}\right)^{1 / n}
\end{aligned}
$$

with

$$
\left|\boldsymbol{r}_{\boldsymbol{i}}-\boldsymbol{r}_{\boldsymbol{j}}\right|=\sqrt{\left(x_{i}-x_{j}\right)^{2}+\left(y_{i}-y_{j}\right)^{2}}
$$

(3) is the correlation function with $\boldsymbol{r}_{i j}$ indicating the measurement location, i.e., pixel coordinates. $u$ represents the correlation parameter related to the correlation length $r_{s}$ given in (4). $r_{s}$ represents the distance with $50 \%$ correlation. The choice of this function is not arbitrary, as it has to fulfill a series of properties (details in Geiger, 1996 or Neyer, 2016). In (3) there are three parameters to be estimated: $\sigma_{s}^{2}, \mathrm{u}$, and $n$. These parameters are computed based on a least-squares adjustment of the autocorrelation of the computed relative displacements between the color channels. The model assumes isotropy, i.e., no directional dependency.

With all parameters of (3) determined (see result section), relative displacements of the red and blue channel with respect to the green can be computed as:

$$
\widehat{\boldsymbol{d}}\left(\boldsymbol{x}^{\prime}, \boldsymbol{y}^{\prime}\right)=A^{\prime} \widehat{\boldsymbol{p}}+C_{\boldsymbol{s}^{\prime} \boldsymbol{s}} \cdot \widehat{\boldsymbol{k}}
$$

$\widehat{\boldsymbol{d}}$ is the estimated displacement positions $\boldsymbol{x}^{\prime}, \boldsymbol{y}^{\prime}$, being the sum of the deterministic and stochastic parts. $\boldsymbol{C}_{\boldsymbol{s} / \boldsymbol{s}}$ gives the link between measured and interpolated pixel coordinates.

\subsection{Photogrammetric Network}

We use Agisoft Metashape (2019) for processing the different image datasets in a standard approach: Image features are detected, matched, and used in bundle adjustment with selfcalibration to create a sparse point cloud. The point cloud was further filtered by points seen in at least three images and with reprojection errors not larger than one pixel. Following this processing, coded targets were detected and the free network was transformed (similarity transformation) onto the geodetic reference frame. Finally, a dense point cloud in its highest resolution with mild filtering was computed for a $2 \times 2 \mathrm{~m}$ section. The procedure was applied to five datasets:

(1) The reference dataset (Lumix images)

(2) The GoPro dataset without CA correction

(3) The green channel of the GoPro dataset

(4) The CA corrected GoPro dataset using the Brown model

(5) The CA corrected GoPro dataset using the collocation model 

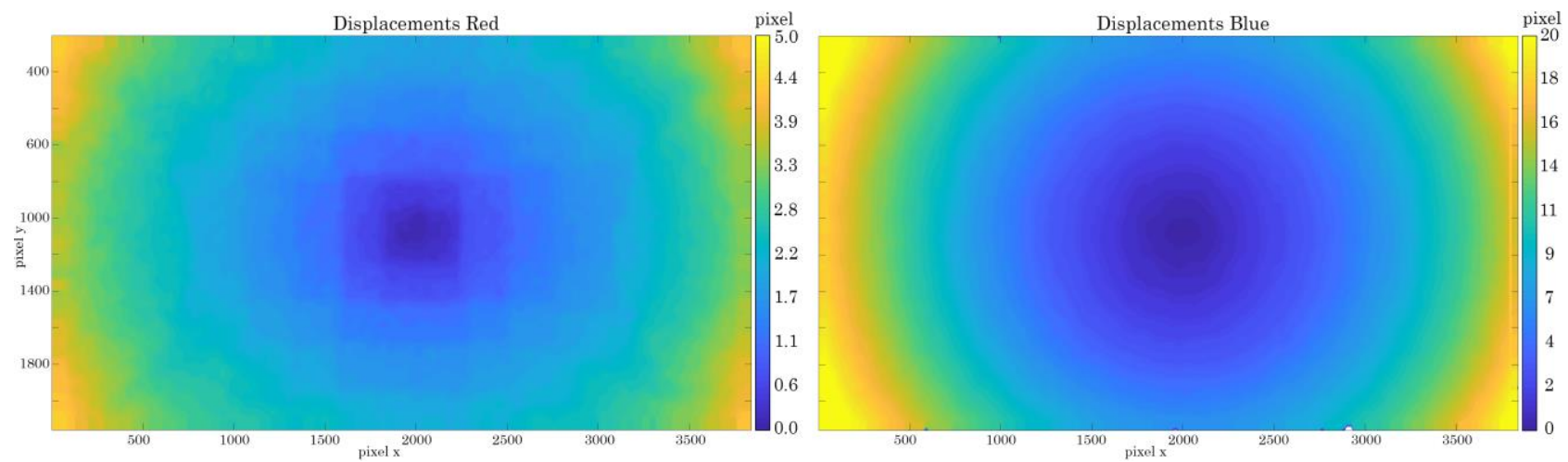

Figure 3. Estimated displacements of red (left) and blue (right) channel features with respect to the green channel. Note the scale difference: a maximum displacement of about 4 pixel was obtained for the red channel, whereas a maximum displacement of more than 20 pixel was found for the blue channel. The block-like structure in the red channel is an effect of the median filtering of all estimated displacements in this dataset.

\subsection{Dense Point Cloud Comparison}

The comparisons of the point clouds is performed in CloudCompare (2017). The dense point clouds are triangulated into polygonal mesh models using the Poisson algorithm implemented in CloudCompare, preserving the original point space resolution (better than $1 \mathrm{~mm}$ ).

The geometric difference between the different models is measured as mesh-to point distance, i.e. distances are computed for each vertex of one model relatively to the polygons of other (reference) mesh.

We compare the differences on two selected areas, i.e., a coral and sandy ocean floor, both in the 2 × $2 \mathrm{~m}$ section (Figure 1).

\section{RESULTS}

\subsection{Reduction of Chromatic Aberration}

Presented here are the solutions obtained for correcting the chromatic aberration with (1) the Brown model parameterization and (2) the collocation approach. The estimated chromatic aberration, i.e., relative displacements of the red and blue channel with respect to the green, are shown in Figure 3.
First, the parameters for the Brown correction model are estimated directly from the estimated displacement components among the channels. Parameters are tested for their significance whereas only the shift parameters and the radial distortion parameters are found to be significant.

Second, the parameters for the correlation function are estimated for both the red and blue channels respectively. A deterministic trend (polynomial of $2^{\text {nd }}$ order) is removed prior to the computation of the autocorrelation. Figure 4 shows that the respective correlations $r_{s}$ are between 300 and 520 pixels for the two channels. An additional feature that can be seen in Figure 3 is the total variance (indicated by the brown data point at $r=0$ ) and the signal variance given by the first parameter of the correlation function, i.e., $\sigma_{s}^{2}$. The closer $\sigma_{s}^{2}$ to the total variance, the less noise can be expected in the vector field to be collocated. Here we see that the red channel has more relative noise contribution, or in other words, the remaining stochastic signal is much weaker compared to the blue channel. The blue channel on the other hand, has a much higher absolute total variance that indicates more residual signal (and noise).

Using equations (2) and (6), the amount of relative displacements are estimated for the red and blue channels respectively.
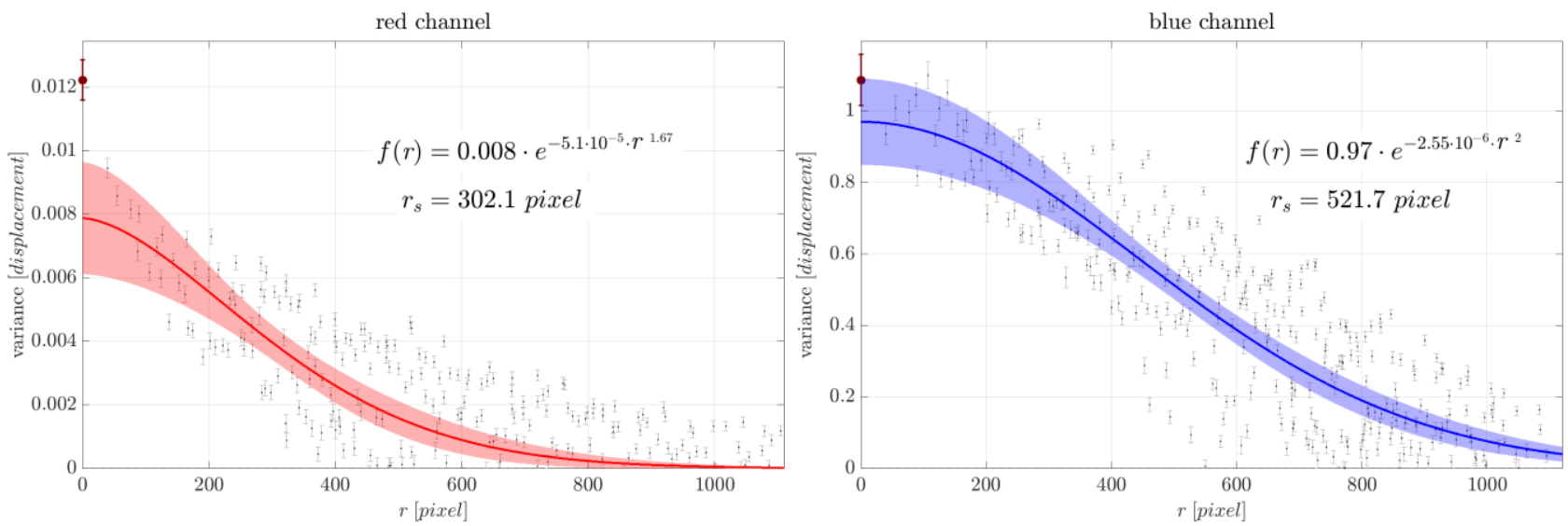

Figure 4. Correlation functions for red (left) and blue (right) channels fitted to the autocorrelation of the displacements. Grey points represent the average autocorrelation between equal distances (with a certain tolerance to gather roughly equal distances). Colored function envelopes show the $3 \sigma$ boundaries of the estimated parameters. Correlation lengths $r_{s}$ are given in pixels. The brown point at $r=0$ indicates the total variance, i.e., $\sigma_{s}^{2}+\sigma_{n}^{2}$. Note the different scales on the ordinate axes. 


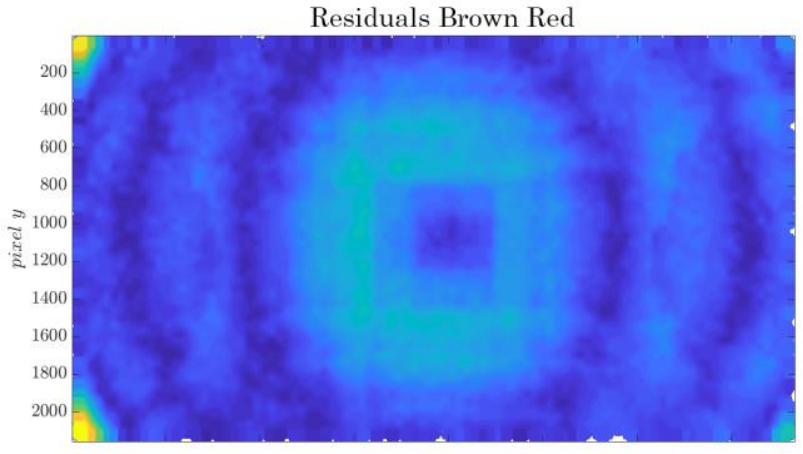

Residuals Brown Blue

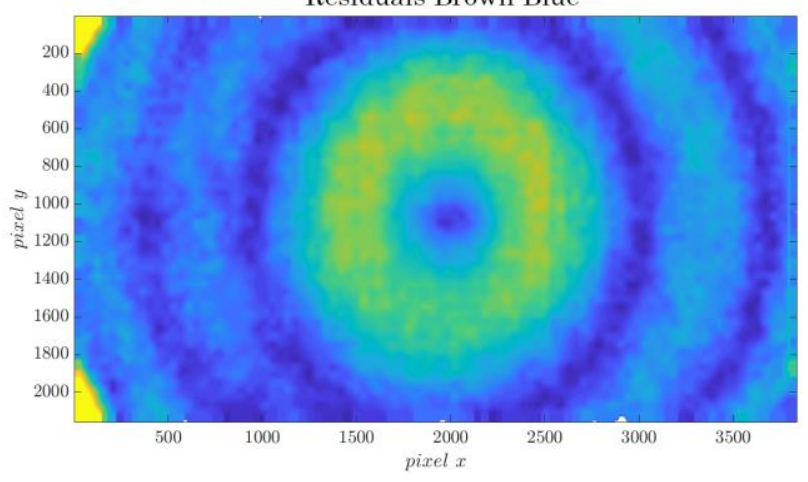

Figure 5. Residual displacement estimates after correcting CA by radial distortion parameters (Brown model) on the left, and with the collocation approach on the right. The top row shows the red channel, the bottom row the blue channel.

Figure 5 shows the residuals obtained for the Brown and collocation models. In case of the Brown model, residuals up to 2 pixel remain, whereas for the collocation model, residuals in the subpixel regime are obtained. The Brown correction model resulted in a posteriori $\sigma_{0}$ of 0.31 and 0.54 pixel for the red and blue channel respectively. Using the collocation approach, the respective a posteriori $\sigma_{0}$ of the red and blue channels were 0.01 and 0.02 pixels. While there is an obvious difference between the two models, practically the corrected images are hard to differentiate. Because our GoPro images have a soft and (in the corners) blurred appearance in general, differences on the level of 1 to 2 pixel cannot be detected visually. An example of the correction effect is shown in Figure 6 with a clear improvement of CA.

\subsection{Photogrammetric Network}

All models are found to perform equally well in the bundle adjustment. Table 2 summarizes the main statistical information from bundle adjustment (as far as provided by Metashape). All
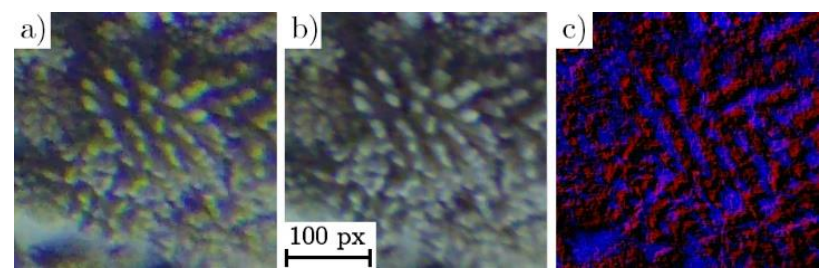

Figure 6. (a) Chromatic aberration seen near the image corner in an underwater GoPro frame. (b) Correction of the CA by relative alignment of the red and blue channel with respect to the green using the standard brown model. (c) Difference between image (a) and (b), slightly enhanced.

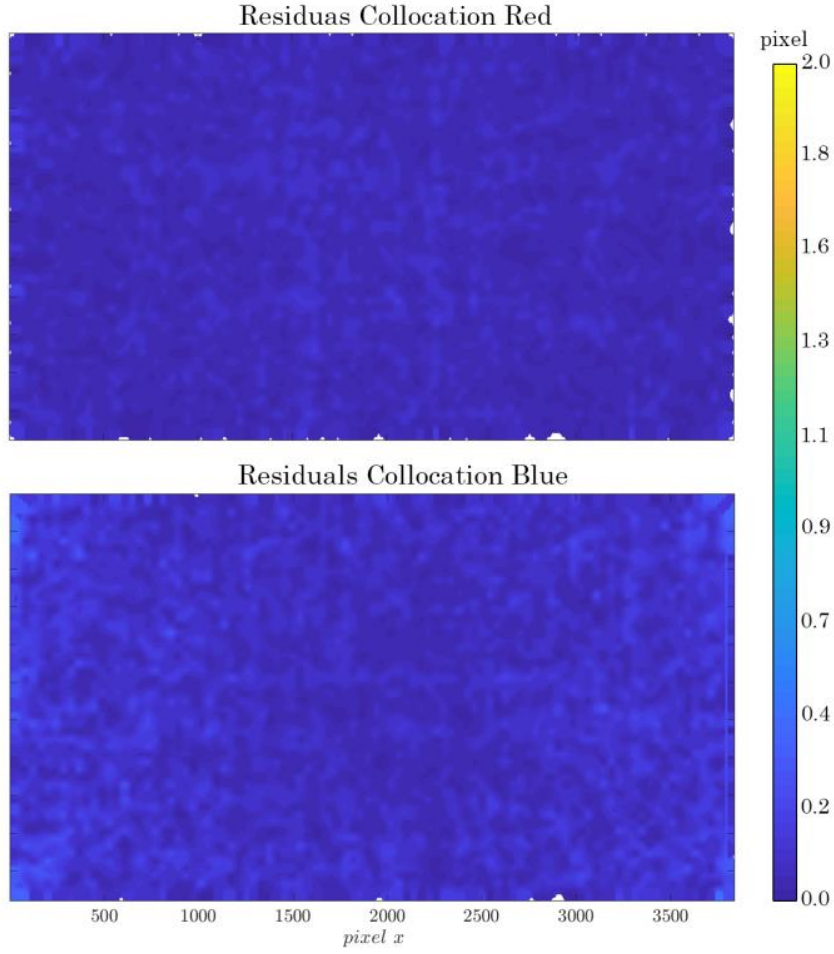

models show consistent RMSE values (definitions can be found in Nocerino et al. 2019). The computation of the dense point clouds in the $2 \times 2 \mathrm{~m}$ area (compare Figure 1) resulted in about 18 Million points for the GoPro models and about 75 Million points for the reference model.

The self-calibration with the Brown model leaves uncompensated systematic errors (see also Nocerino et al., 2019). If we compute the standard deviations of the object space coordinates, as done in Nocerino et al., 2019, the Lumix results turn out better by a factor of 1.5 in planimetry and 2 in height, what was to be expected.

\subsection{Dense Point Cloud Comparisons}

In contrast to the empirical results obtained from bundle adjustment, obvious differences exist between the dense point clouds of the different datasets. We select two representative regions to illustrate the results in more detail.

\begin{tabular}{|c|c|c|c|c|}
\hline & $\begin{array}{c}\text { \# tie } \\
\text { points }\end{array}$ & RMSExy & RMSEz & RMSE3D \\
\hline Reference & $3366^{\prime} 627$ & 3.8 & 2.3 & 5.8 \\
\hline GoPro_1 & $303^{\prime} 151$ & 3.8 & 3.0 & 6.2 \\
\hline GoPro_2 & $306 ' 546$ & 3.8 & 2.8 & 6.1 \\
\hline GoPro_3 & $301^{\prime} 697$ & 3.8 & 2.9 & 6.2 \\
\hline GoPro_4 & $302^{\prime} 429$ & 3.9 & 3.0 & 6.2 \\
\hline
\end{tabular}

Table 2. Performance during bundle adjustment. GoPro_1 = without CA correction, GoPro_2 = only green channel,

GoPro_3 = CA corrected using Brown model, GoPro_4 = CA corrected using collocation model. RMSE values in millimeter. 
a)

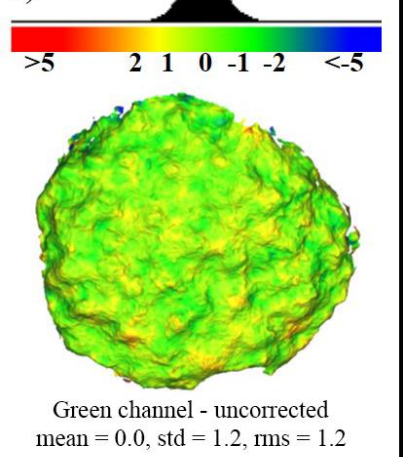

b)
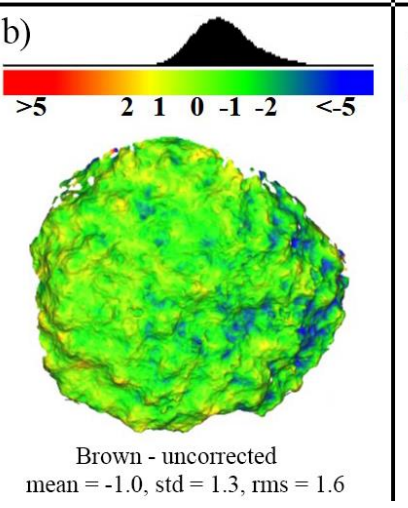

c)
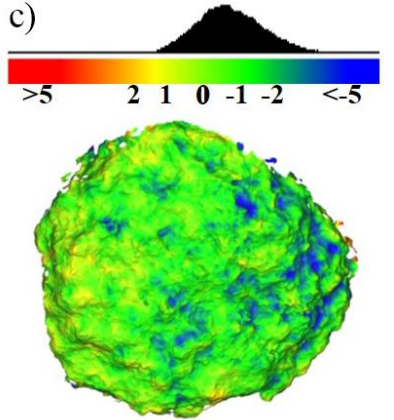

Collocation - uncorrected mean $=-1.2, \mathrm{std}=1.3, \mathrm{rms}=1.8$

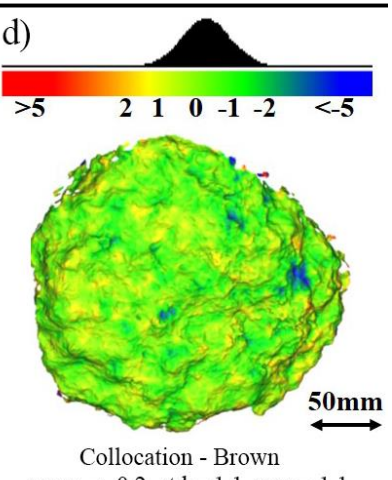

mean $=-0.2, \mathrm{std}=1.1, \mathrm{rms}=1.1$

Figure 7. Comparison of dense point clouds for a single coral. a) to c) compare models generated by the green channel, the Brown dataset, and the collocated dataset with the uncorrected GoPro dataset. In d) the difference between the collocation and

Brown datasets are shown. All numbers in millimeters.

\section{Coral}

Figure 7 shows the differences obtained for a (living) coral. The coral image is shown in Figure 8. Among the presented discrepancies (mapped on the respective meshes), there are systematic variations of up to $1.2 \mathrm{~mm}$ with standard deviations of $1.3 \mathrm{~mm}$. Because the geometrical setting is identical in all cases presented in Figure 7, these differences can directly be related to the effects of the color channels and there CA. Using only the green channel for building the model seems to result in steeper gradients that can be observed as positive differences on coral branches (Figure 7a). Point clouds based on the collocated images as well as the images corrected by the Brown model show similar differences with respect to the uncorrected dataset (Figure $7 \mathrm{~b}$ and $7 \mathrm{c}$ ). The difference among the latter two also has a standard deviation in the order of $1 \mathrm{~mm}$ (Figure 7c).

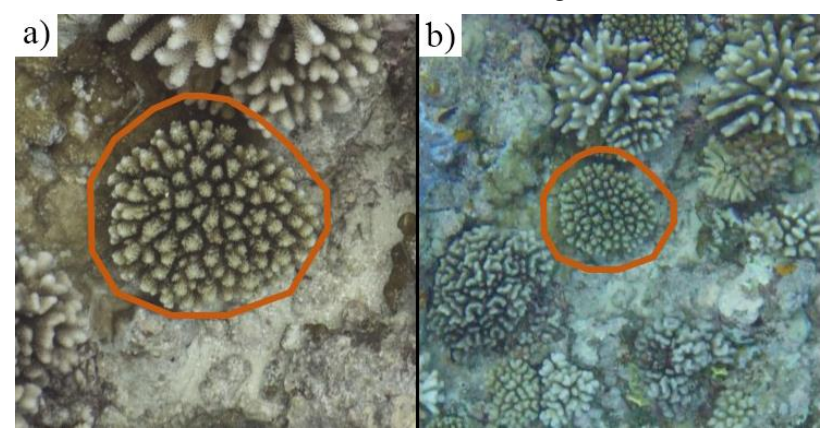

Figure 8. Selected coral used to compare the different models. a) shows a sample image from the reference camera and b) a sample image from the GoPro camera.

a)

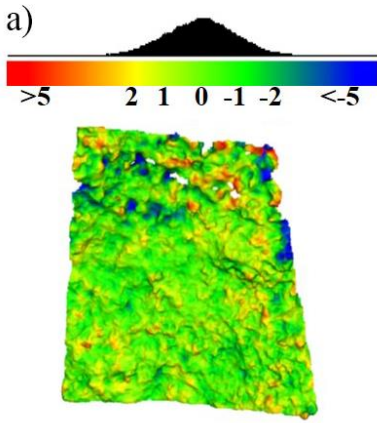

Green channel - uncorrected
mean $=0.3, \mathrm{std}=1.4, \mathrm{rms}=1.4$
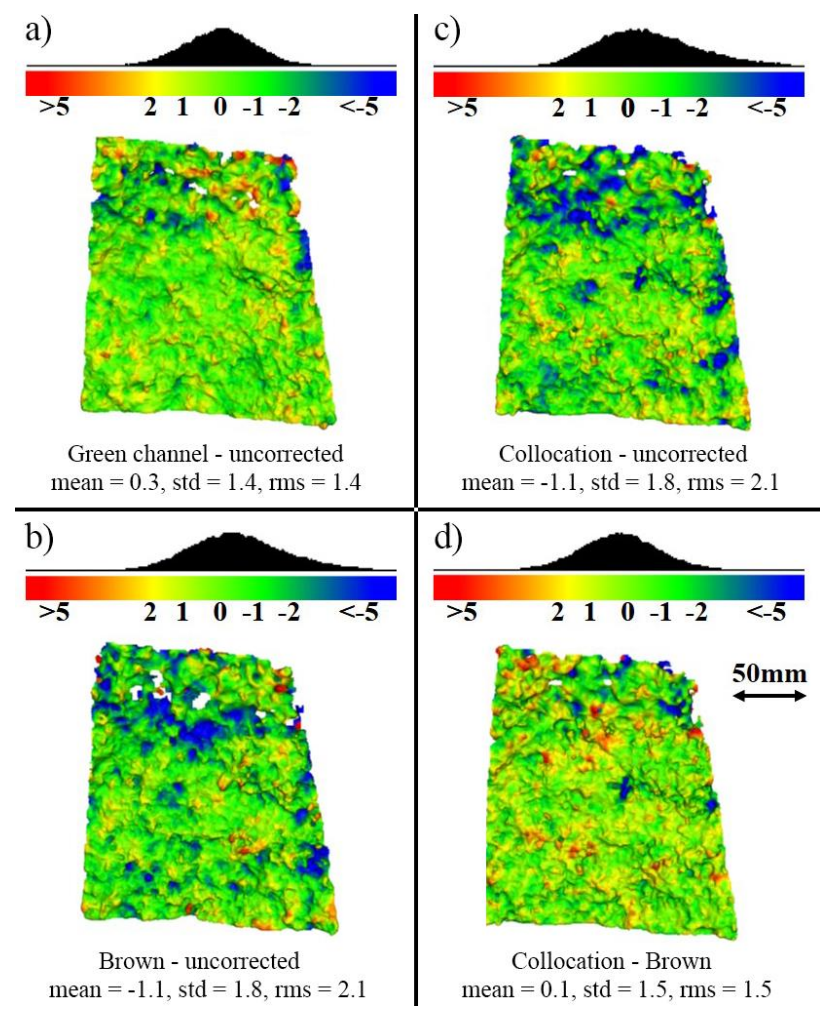

Collocation - uncorrected mean $=-1.1, \mathrm{std}=1.8, \mathrm{rms}=2.1$

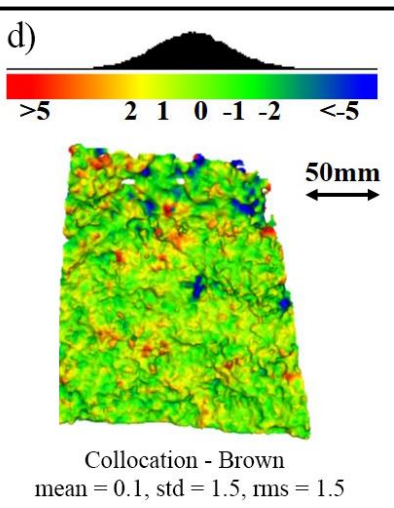

Figure 9. Comparison of dense point clouds for an ocean floor area. As in Fig.7, a) to c) compare models generated by the green channel, the Brown dataset, and the collocated dataset

with the uncorrected GoPro dataset. In d), the difference between the collocation and Brown datasets are shown. All numbers in millimeters.

\section{Ocean Floor}

Differences between the point clouds for a model section with mostly ocean floor is given in Figure 9. The area is within the blue rectangle seen in Figure 1. Because the entire model is a densely populated coral area, the ocean floor section is not flat but rather characterized by an accumulation of various items (sand, rocks, dead coral debris, etc.). As in case of the coral comparison, a similar pattern is visible, whereas there are more areas of extreme differences ( $\geq 5 \mathrm{~mm}$ colored in red, $\leq-5 \mathrm{~mm}$ colored in blue). Again, the results of the two correction models show some level of agreement. The point cloud generated using the collocated images shows higher extremes: Positive differences are primarily seen on top of model peaks whereas negative differences are located mostly in valleys (Figure 9c).

\section{Comparison with the reference model}

While comparisons among the GoPro datasets with different correction models only show differences due effects of the color channel combinations, a comparison with our reference model turned out to be difficult for interpretation (Figure 10). Although the reference dataset has better image quality and a smaller GSD, the photogrammetric network is also different: Due to the nature of acquisition, images were not captured at the same location, with the same orientation, and the same field of view. Consequently, differences of more than $5 \mathrm{~mm}$ are visible, especially near vertical structures (side of coral, Figure 10a and 
a)

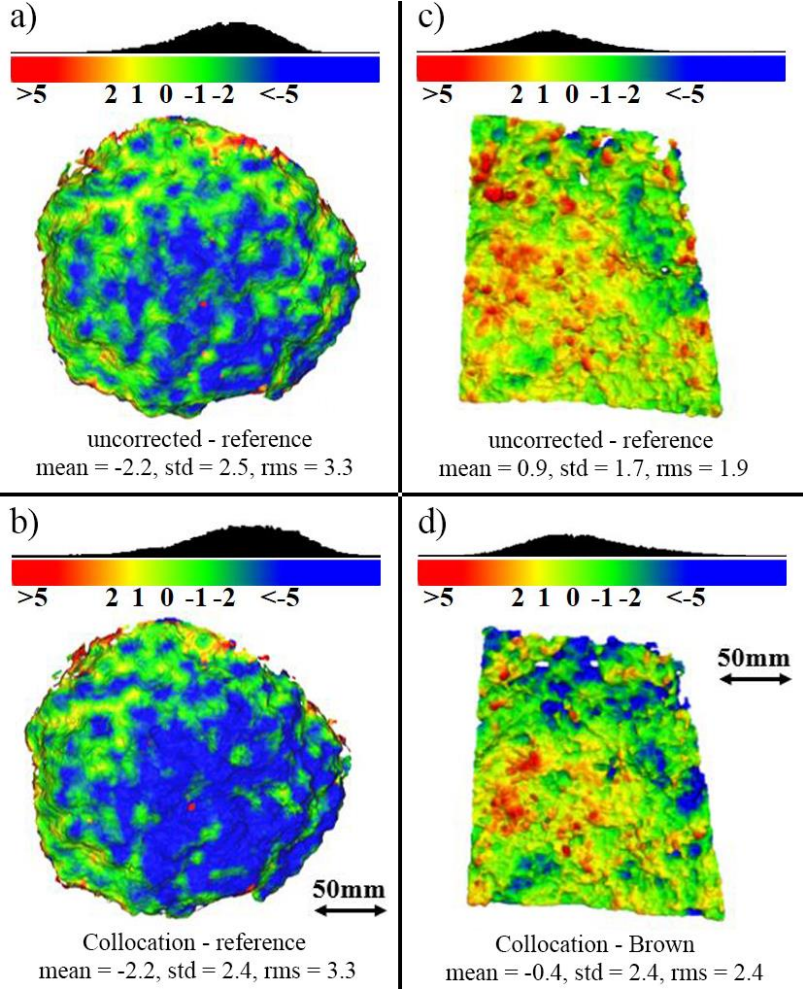

a)

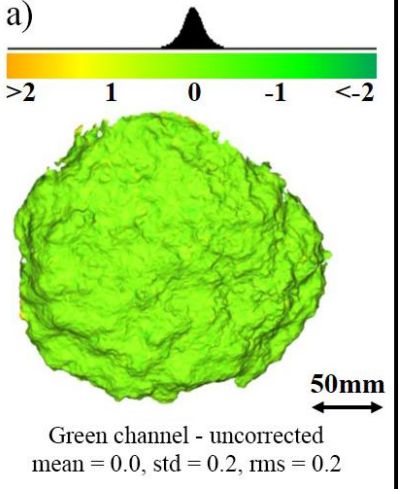

b)

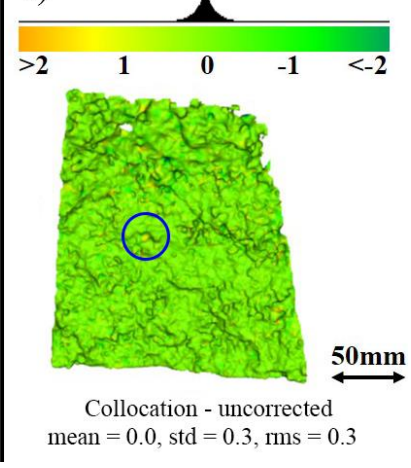

Figure 11. Comparison of point clouds generated by repeating the dense image matching procedure for a) the selected coral, and $b$ ) the ocean floor. The blue circle in b) highlights an area

where deviations in the order of $1 \mathrm{~mm}$ can be observed.

expectations (standard deviations of object space coordinates) could not be reached. We are still working on this issue.

In the second part, the study presented the effects of CA correction on the resulting 3D models. Interestingly, no significant difference in the bundle adjustment between the different correction methods could be observed. With an average GSD of $1.2 \mathrm{~mm}$, a CA correction of 10 pixel (average in the outer areas of the blue channel), implies a shift of $12 \mathrm{~mm}$ in object space. This however is only true for image border areas in the blue channel. Because image quality towards the image borders is severely degraded anyway, most contrast information used to detect and match tie points may be retrieved from the green and red channels. The exact procedure, however, is not accessible.

For dense image matching, the situation looks different: Model deviations in the order of a few millimeters can be observed at various locations. Judgement of which model is closer to reality turned out to be difficult as there is no real known ground truth. The comparison with our (photogrammetric) reference model shows similar deviations with both, the uncorrected and the corrected models. One of the principal problems in dense image matching is the unknown uncertainty involved in the generated point clouds. As such, we cannot judge the quality of dense point clouds due to the improvements of image quality (and contrast) directly. The main differences visible in Figure 10 are mostly related to the difference in resolution (finer details result in higher model peaks or deeper valleys), image contrast (less smoothing between peaks and valleys), and acquisition geometry (different coverage). In addition, there is also an uncertainty in the generation of the dense point cloud itself: when comparing two dense point clouds generated from an identical processing stage (a simple re-computation), results are not identical (see Figure 11). Although deviations are in the sub-millimeter range, deviations of up to $1 \mathrm{~mm}$ can be observed at some isolated locations.

While it remains unclear which GoPro dataset proves to be the most accurate representation of the object space, deviations in the order of four times the GSD were found at sharp object edges. We therefore conclude that the presence of CA significantly influences the dense point clouds, i.e., the estimated 3D models.

In summary, the achievement of very high (subpixel) accuracies of underwater photogrammetry, comparable with "in air" 
applications, seems not to be possible, at least not at present. There are many factors responsible for this fact. Man and equipment is not made for underwater work. Nevertheless, photogrammetry can play an important role in Ocean Science in different ways, if applied with expertise and with a realistic sense of what is possible.

\section{ACKNOWLEDGEMENTS}

This material is based upon work supported by the U.S. National Science Foundation under Grant No. OCE 16-37396 (and earlier awards) as well as a generous gift from the Gordon and Betty Moore Foundation. Very crucial was the financial and scientific support provided by Prof. Matthias Troyer through the Institute of Theoretical Physics, ETH Zurich. The research was executed under permits issued by the French Polynesian Government (Délégation à la Recherche) and the Haut-Commissariat de la République en Polynésie Francaise (DTRT) (Protocole d'Accueil 2005-2018). This work represents a contribution of the Moorea Coral Reef (MCR) LTER Site. The authors thank the Gump Station team, especially Dr. A. Brooks, for their fundamental support in the field missions and useful discussions. We also thank Prof. A. Capra and Dr. F. Menna for their substantial contribution during field work.

\section{REFERENCES}

Brown, D.C., Close-range camera calibration, 1971. Photogramm. Eng. 37 (8), 855-866

CloudCompare, 2017. http://www.cloudcompare.org/, v. 2.9.1.
Farneback, G., 2003. Two-Frame Motion Estimation Based on Polynomial Expansion. Proceedings of the 13th Scandinavian Conference on Image Analysis. Gothenburg, Sweden, 363-370

Geiger, A., 1996, Verzerrungs-Analyse, Interpolation und Approximation, Tech. rep., ETH Zürich

Guo, T., Capra, A., Troyer, M., Grün, A., Brooks, A.J., Hench, J.L., Schmitt, R.J., Holbrook, S.J. and Dubbini, M., 2016. Accuracy assessment of underwater photogrammetric three dimensional modelling for coral reefs. International Archives of the Photogrammetry, Remote Sensing \& Spatial Information Sciences, 41.

Guillaume, S., Muller, C., and Cattin P.-H. Trinet+, logiciel de compensation $3 D$ version 6.1, Mode d'emploi, HEIG-VD, Yverdon, Switzerland, 2008.

Moritz, H., 1970. Least-squares estimation in physical geodesy, Veröffentlichungen der Deutschen Geodätischen Kommission.

Moritz, H. 1973. Least-squares collocation, Deutsche Geodätische Kommission, Reihe A: Theoretische Geodäsie, 75, $1-91$.

Neyer, F., 2016. Monitoring Rock Glaciers by Combining Photogrammetric and GNSS-Based Method, ETH Zürich, 178 p., Doctoral Thesis, 10.3929/ethz-a-010865360

Neyer, F., Nocerino, E. and Gruen, A., 2018. Monitoring coral growth-the dichotomy between underwater photogrammetry and geodetic control network. International Archives of the Photogrammetry, Remote Sensing \& Spatial Information Sciences, 42(2).

Nocerino, E., Neyer, F., Gruen, A., Troyer, M., Menna, F., Brooks, A., Capra, A., Castagnetti C., Rossi, P. 2019: Comparison of Diver-Operated Underwater Photogrammetric Systems for Coral Reef Monitoring. In press 